\title{
Editorial
}

\section{Experimental and Theoretical Advances in Amorphous Alloys}

\author{
Na Chen, ${ }^{1}$ Limei Xu, ${ }^{2}$ Yang Shao, ${ }^{1}$ and Konstantinos Georgarakis ${ }^{3}$ \\ ${ }^{1}$ School of Materials Science and Engineering, Tsinghua University, Beijing 100084, China \\ ${ }^{2}$ International Center for Quantum Materials, Peking University, Beijing 100084, China \\ ${ }^{3}$ WPI Advanced Institute for Materials Research, Sendai 9808577, Japan \\ Correspondence should be addressed to Na Chen; chennadm@mail.tsinghua.edu.cn
}

Received 2 September 2014; Accepted 2 September 2014; Published 31 December 2014

Copyright (C) $2014 \mathrm{Na}$ Chen et al. This is an open access article distributed under the Creative Commons Attribution License, which permits unrestricted use, distribution, and reproduction in any medium, provided the original work is properly cited.

Unlike crystalline alloys having long-range order in atomic arrangements, amorphous alloys are disordered solids [1-4]. Yet their structure when solidified is not completely disordered but contains short- to medium-range ordered clusters $[5,6]$. This unique structure endows them with superior mechanical, physical, and chemical properties. Therefore, amorphous alloys are of both scientific and technological interests. Recent progress in experimental and computational techniques has greatly advanced our understanding of a number of key issues such as glass structure at atomic level, glass-forming ability (GFA), and structure-property relationship in this field $[7,8]$. In spite of this, ongoing research efforts remain necessary to uncover the atomiclevel mechanisms underlying the glass formation and the deformation, as well as the glass structure.

Bulk amorphous alloys (BAAs) with diameters of more than one centimeter are preferred for practical engineering applications. To develop such alloy systems with extremely high GFA, several strategies, including large undercooling (LU), Inoue's three empirical rules (ITER), and flux treatment, have been proposed [2, 9-11]. Among them, minor alloying additions (MAA) and fluxing prove to be significantly beneficial for enhancing GFA and thus enabling the formation of centimeter-scale BAAs [12-14]. As listed in Table 1, a combination of these strategies could be employed for producing centimeter-scale BAAs.

In addition to glass formation, the mechanical property of amorphous alloys is also one of the hot topics in this field. Although shear localization is responsible for their catastrophic failure, there is a need for a better understanding of initiation, development, and percolation of these shear transformation events. At atomic level, shear transformation zones (STZs) are supposed to be activated in response to external stimuli and eventually develop into shear bands during deformation. Unfortunately, these clusters are very small with an average size of $\sim 1 \mathrm{~nm}$ and appear only at a very early stage of deformation, which makes it very difficult to be observed experimentally. On the other hand, both structural disordering and the formation of nanocrystals have been evidenced inside shear bands after deformation [15], implying that atomic rearrangements must occur. Moreover, the nanocrystal size and their distributions could be related to shear transformations, particularly when the energy barrier to crystallize is comparable to or even smaller than that to activate percolated STZs globally. As a consequence of deformation, structural evolution inside shear bands could help uncover the underlying deformation mechanisms for amorphous alloys.

Last but not least, dealloying amorphous alloys has raised tremendous attentions by producing highly active nanoporous metals used for catalysts and electric devices such as solar cells, sensors, or some other electric components. This provides a convenient way to design novel nanostructured materials with desirable functionalities.

In summary, this special issue is mainly focused on the development of bulk amorphous alloy, mechanical response, and new functionalities explored in amorphous alloys. We hope that the readers will find in this special issue not only interesting experimental results on the formation of centimeter-scale BAAs induced by fluxing or minor alloy additions and new functional nanoporous metals by dealloying amorphous alloys and amorphous alloy composites 
TABLE 1: The strategies for producing centimeter-scale amorphous alloys.

\begin{tabular}{lcccc}
\hline Representative BAAs & Critical size for glass formation $(\mathrm{mm})$ & LU & ITER & Fluxing \\
\hline Pd-Ni-Cu-P [16] & 80 & $\sqrt{ }$ & $\sqrt{ }$ & $\sqrt{ }$ \\
Zr-Ti-Cu-Ni-Be [17] & 50 & $\sqrt{ }$ & $\sqrt{ }$ & $\sqrt{ }$ \\
Cu-Zr-Al-Ag [16] & 30 & $\sqrt{ }$ & $\sqrt{ }$ & $\sqrt{ }$ \\
Fe-Cr-Co-Mo-Mn-C-B-Y [18] & 12 & $\sqrt{ }$ & $\sqrt{ }$ & $\sqrt{ }$ \\
\hline
\end{tabular}

combining with high strength and large ductility but also theoretical advances in the underlying deformation mechanisms at atomic scale.

\section{$\mathrm{Na}$ Chen \\ Limei Xu \\ Yang Shao \\ Konstantinos Georgarakis}

\section{References}

[1] A. L. Greer, "Metallic glasses," Science, vol. 267, no. 5206, pp. 1947-1953, 1995.

[2] A. Inoue, "Stabilization of metallic supercooled liquid and bulk amorphous alloys," Acta Materialia, vol. 48, no. 1, pp. 279-306, 2000.

[3] W. L. Johnson, "Bulk glass-forming metallic alloys: science and technology," MRS Bulletin, vol. 24, no. 10, pp. 42-56, 1999.

[4] W. H. Wang, C. Dong, and C. H. Shek, "Bulk metallic glasses," Materials Science and Engineering R: Reports, vol. 44, no. 2-3, pp. 45-90, 2004.

[5] D. B. Miracle, "A structural model for metallic glasses," Nature Materials, vol. 3, no. 10, pp. 697-702, 2004.

[6] H. W. Sheng, W. K. Luo, F. M. Alamgir, J. M. Bai, and E. Ma, "Atomic packing and short-to-medium-range order in metallic glasses," Nature, vol. 439, no. 7075, pp. 419-425, 2006.

[7] A. Hirata, P. Guan, T. Fujita et al., "Direct observation of local atomic order in a metallic glass," Nature Materials, vol. 10, no. 1, pp. 28-33, 2011.

[8] Y. Q. Cheng and E. Ma, "Atomic-level structure and structureproperty relationship in metallic glasses," Progress in Materials Science, vol. 56, no. 4, pp. 379-473, 2011.

[9] M. Marcus and D. Turnbull, "On the correlation between glassforming tendency and liquidus temperature in metallic alloys," Materials Science and Engineering, vol. 23, no. 2-3, pp. 211-214, 1976.

[10] H. W. Kui, A. L. Greer, and D. Turnbull, "Formation of bulk metallic-glass by fluxing," Applied Physics Letters, vol. 45, no. 6, pp. 615-616, 1984.

[11] N. Chen, K. Yao, and F. Ruan, "Influence of flux treatment on the glass forming ability of Pd-Si binary alloys," Journal of University of Science and Technology Beijing, vol. 14, no. 1, pp. 4-7, 2007.

[12] Z. P. Lu and C. T. Liu, "Role of minor alloying additions in formation of bulk metallic glasses: a review," Journal of Materials Science, vol. 39, no. 12, pp. 3965-3974, 2004.

[13] W. H. Wang, "Roles of minor additions in formation and properties of bulk metallic glasses," Progress in Materials Science, vol. 52, no. 4, pp. 540-596, 2007.

[14] N. Chen, L. Martin, D. V. Luzguine-Luzgin, and A. Inoue, "Role of alloying additions in glass formation and properties of bulk metallic glasses," Materials, vol. 3, no. 12, pp. 5320-5339, 2010.
[15] A. L. Greer, Y. Q. Cheng, and E. Ma, "Shear bands in metallic glasses," Materials Science and Engineering R: Reports, vol. 74, no. 4, pp. 71-132, 2013.

[16] A. Inoue and A. Takeuchi, "Recent development and application products of bulk glassy alloys," Acta Materialia, vol. 59, no. 6, pp. 2243-2267, 2011.

[17] W. L. Johnson, "Bulk amorphous metal-an emerging engineering material," JOM, vol. 54, no. 3, pp. 40-43, 2002.

[18] Z. P. Lu, C. T. Liu, J. R. Thompson, and W. D. Porter, "Structural amorphous steels," Physical Review Letters, vol. 92, no. 24, Article ID 245503, 2004. 

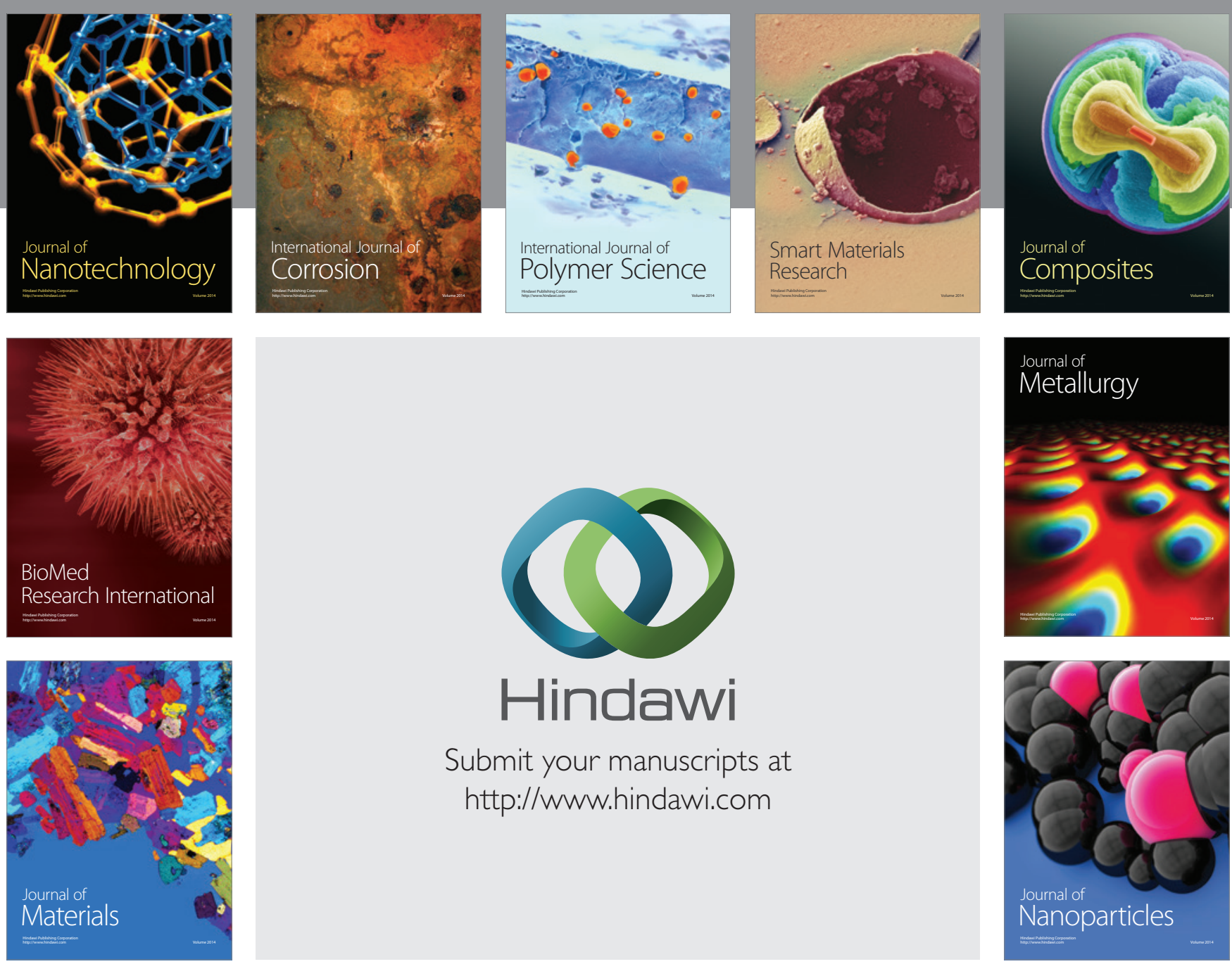

Submit your manuscripts at http://www.hindawi.com
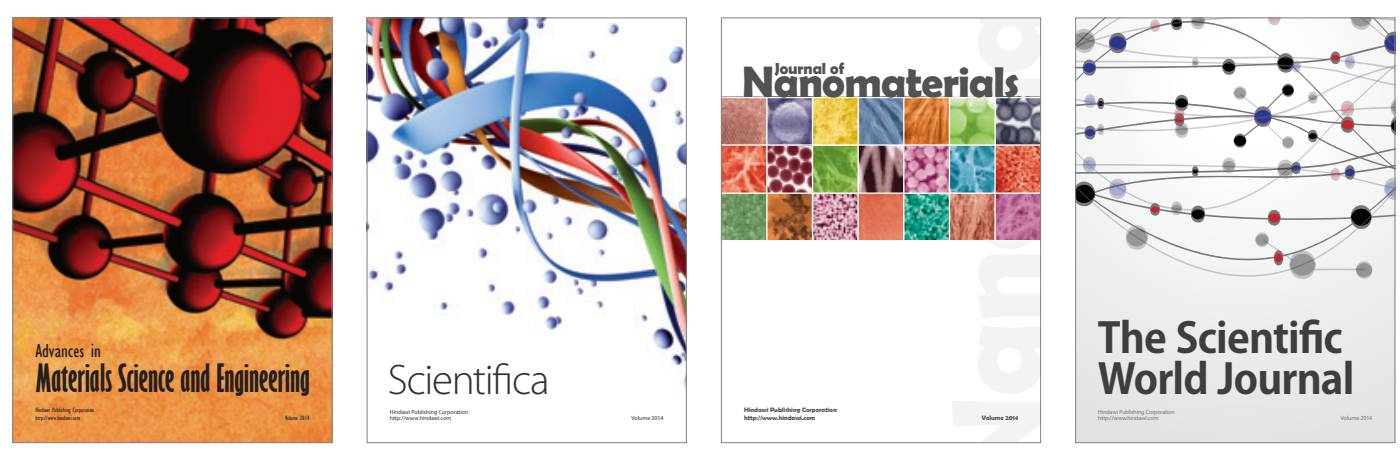

\section{The Scientific World Journal}
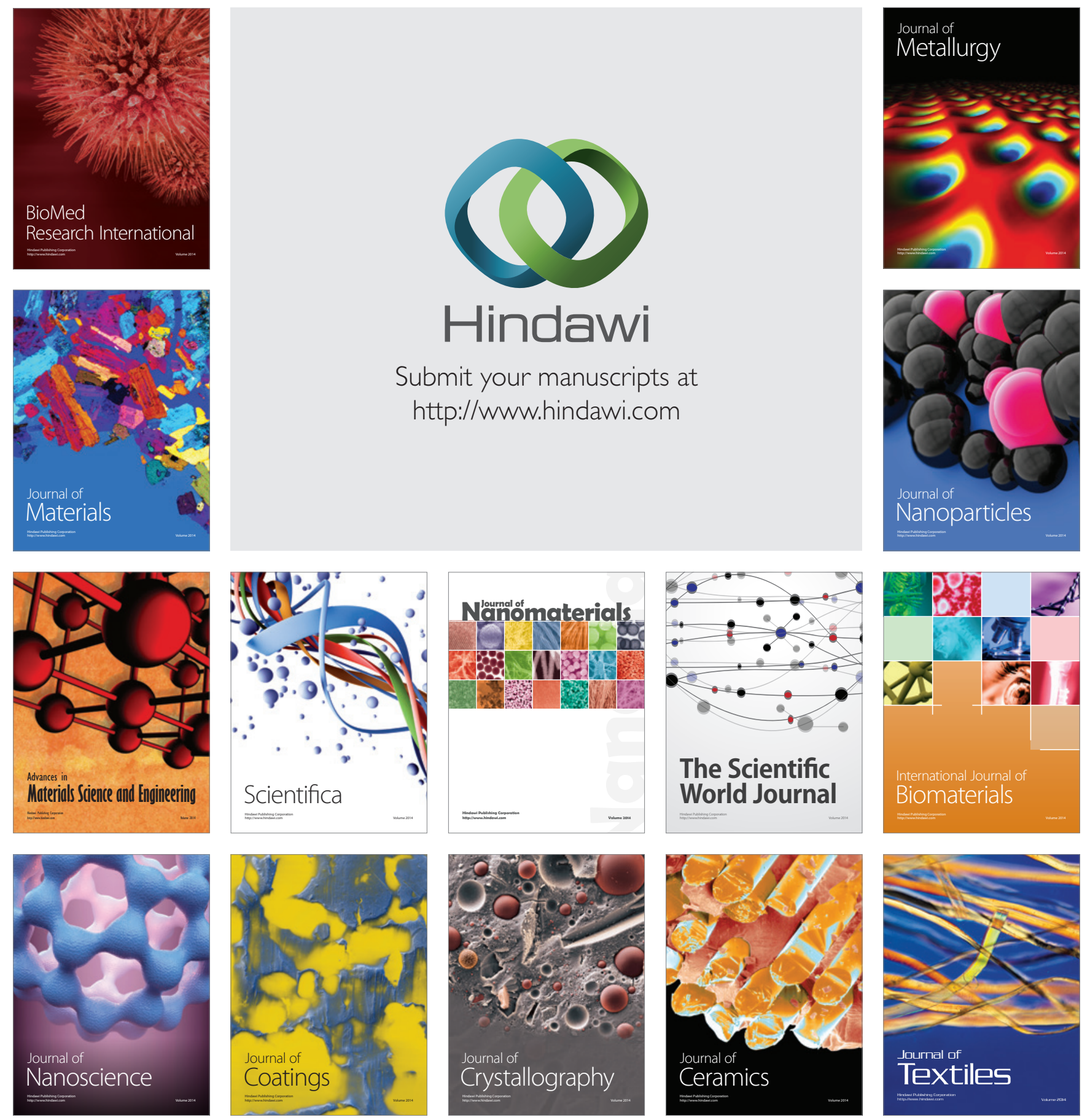